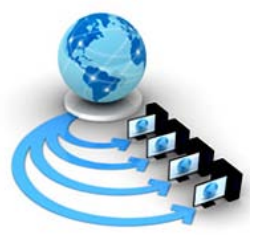

Volume 9, No. 2, March-April 2018

International Journal of Advanced Research in Computer Science

REVIEW ARTICLE

Available Online at www.ijarcs.info

\title{
LIGHT FIDELITY: A WAY OF DATA TRANSMISSION
}

Osheen Sharma

Assitant Professor

Department of Information Technology

GGDSD College

Chandigarh (UT), India
Shruti

Assitant Professor

Department of Information Technology

GGDSD College

Chandigarh (UT), India

\begin{abstract}
Li-Fi is a very efficient, simple and cheaper network that can be used for wireless data transmission. It is considered to be better than the trending technology, i.e. Wi-Fi, in terms of cost and efficiency of usage. Li-Fi complies with the IEEE standard 802.15.7. The paper focuses on explaining the concept and meaning of Li-Fi technology. In this paper we have explained the different components necessary for constructing a Li-Fi system. Its features, different applications and future uses are also discussed in brief. The use and importance of LED light in the working of Li-Fi is also described. The VLC technology (for increasing the transmission speed) used in Li-Fi has been explained briefly.
\end{abstract}

Keywords: Li-Fi; Light Fidelity; VLC; Wi-Fi; LED; wireless communication; data transmission

\section{INTRODUCTION}

In this era of data communication where transmission of data is one of the most important activity taking place using different mediums, Li-Fi is a new medium to do so. Li-Fi uses LED (light emitting diode) light for transmitting data wirelessly. The current wireless network connects the user to the internet that helps in data transmission to take place between different sites using the Wi-Fi technology. Wi-Fi is the wireless network that transmits data from one site to another using the internet services. This wireless system of data transmission is very slow when it comes to connect various devices together. The fixed bandwidth of internet available is the main reason of its slow speed, which makes it more difficult to transfer high speed data.

The LED bulb when first developed in early 1960. It was a high brightness blue LED demonstrated by Shuji Nakamura of Nichia Corporation in 1994. This led to the development of white LED bulb by Nakamura, Isamu Akasaki and Hiroshi Amano [2]. No one thought this white LED would serve any other purpose in the future other that lighting the world that too transmit data.

Li-Fi was first introduced by a German physicist Harald Hass in the TED (Technology, Entertainment, and Design) Global talk on Visible Light Communication (VLC) in July 2011. He referred to it as "data through illumination" and used a table lamp with an LED bulb to transmit a video of a blooming flower that was then projected onto a screen. It was Heral Hass, a German physicist who really thought about using Visible Light Communication for data transmission. He first talked about it in July 2011 in TED (Technology, Entertainment, and Design) Global Talk. He demonstrated the use of LED light for transmission by using a LED lamp for transferring a video on the projector screen [1].

Harald gave a new use of LED bulbs to the world (except lighting the room) by performing this experiment and making it successful. He made sure that everyone believes that it is the light bulb which is projecting video on the screen. The basic idea of introducing the Li-Fi technology is to enjoy a high speed data transmission that can take place by just using the LED lights.

According to Hass invention, which he called as "D-Light", it can produce data rate faster than 10 megabits per second
(Mbps). It is faster than an average broadband connection, even Wi-Fi network.

\section{A. Features of Li-Fi}

- Data transmission solution to cellular and Wi-Fi In homes and offices where mobile and $\mathrm{Wi}-\mathrm{Fi}$ networks draw a weaker signal, $\mathrm{Li}-\mathrm{Fi}$ is a viable alternative for high bandwidth as it can handle congestion which is created by Wi-Fi.

- Wireless hotspots in dense urban areas

The scarcity of radio waves for transmitting data is very much present in metropolitan areas and mega cities. Radio waves are widespread and can cause security problems with Radio frequency signals. Whereas Li-Fi uses multiple light sources (LED bulbs) and they increase the network security and data transmission rate.

- Multimedia consumption

$\mathrm{Li}-\mathrm{Fi}$ is best for transmitting live streaming video and substantial downloads as it delivers excellent downlink speeds without the need to uplink too much data back.

- Charging mobile devices

$\mathrm{Li}-\mathrm{Fi}$ is good for both for information transmission and conjointly for the charging of electronic devices (like mobile phones, tablets and wristwatches). The structure includes a thin crystal layer incorporated into the show that acts like solar photoreceptor.

- A more efficient airplane entertainment and internet solution

Li-Fi by using LED reading bulb present inside airplanes provides a better, faster and cheaper means of communication to passengers travelling in an aircraft as compared to Wi-Fi which provides low speed internet at a very high cost. Also, there are chances that Wi-Fi interferes with the navigational systems of the pilots.

- Context-aware, location-based services

$\mathrm{Li}$-Fi enabled Smartphone and mobile device users can receive relevant information to their locations. It introduces many promotional and navigational opportunities for organizations and confluence. 


\section{WORKING OF LI-FI TECHNOLOGY}

At present, Li-Fi is the fastest medium for information exchange in wireless communication network. It uses VLC (visible light communication) data communication medium which needs visible light of range $400 \mathrm{THz}-800 \mathrm{THz}$ as a mean to transmit data. Basically, fats pulses of light are used for data transmission through wireless channel.

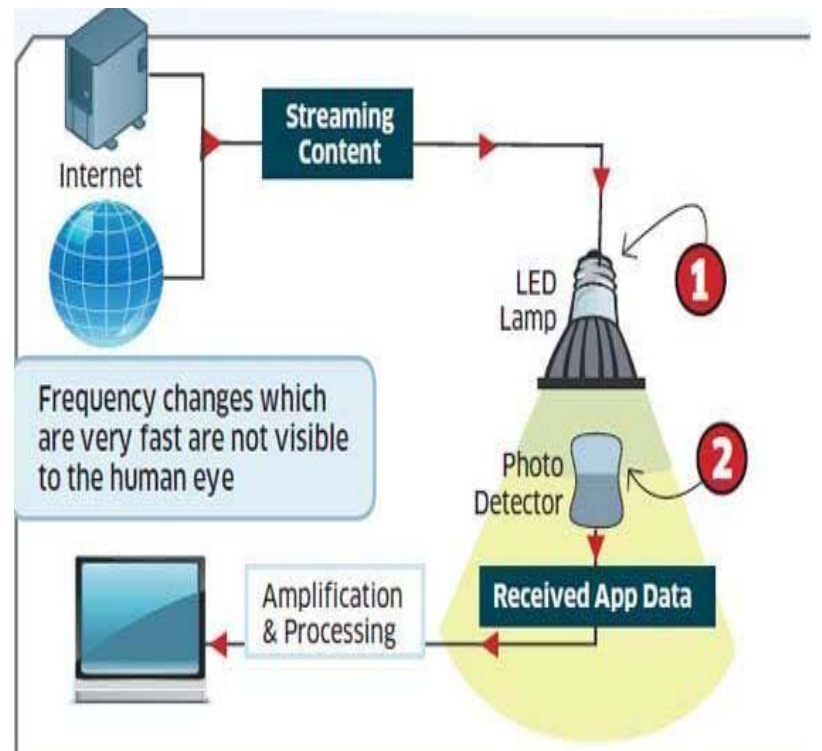

Figure 1. Block diagram of Li-Fi system [1]

LED generates digital strings, as they can be switched on and off. There is LED light on one end and a photo-detector on the other end. If the LED is on, the photo detector (light sensor) registers a binary one; otherwise it's a binary zero. When LED is flashed multiple times, a message is generated. With the arrangement of LEDs and some different colors, a data rate of 100 or more Mbps can be achieved. This results in a speed of 10Gbps (approximately) which is sufficient to download a High definition movie in just 30secs.

Internet connection, switch and LED lamp are all connected to the lamp driver through optical fiber cable. Photo detector receives the signal and performs further operations. Detector is further connected to PC's, Laptops or LAN port. When the LED is ON, the conversion of the digital data into the light form is done by microchip. On receiving the light signal the Light detector converts it again into the original digital form [3].

\section{IMPLEMENTATION}

\section{A. Components of Li-Fi system:}

There are mainly two components which are required for setting up the Li-Fi system. These are:

- A high brightness white LED which acts as transmission source.

- A silicon photodiode with good response to visible light as the receiving element.

Data when sent is converted into byte format, the intermediate data representation and then the light signals are emitted by the transmitter after conversion. The photo diode on the receiver side then receives the light signals. Similarly, a reverse process is performed in order to get back the data from the light received.

\section{B. Benefits of $\mathrm{Li}-\mathrm{Fi}$}

- Capacity: The bandwidth is increased in Li-Fi. The issue of shortage of spectrum to some extent is resolved in $\mathrm{Li}-\mathrm{Fi}$ because it is far wider. The spectrum is inflated by 10,000 times than radio waves [4].

- Efficiency: Data transmission using Li-Fi is very cheap as it consumes less energy and is more efficient.

- Availability: LED bulbs are available everywhere (home, office and malls, etc.) and where there is availability of light bulbs, data transmission is possible using Li-Fi.

- Security: One of the major concerns in data transmission is security and one of the main advantages of Li-Fi is high security. Since light cannot pass through opaque structures, Li-Fi Internet is available only to the users within a room and cannot be breached by users in other rooms or building [5].

- $\quad$ Cost effective: As it requires very less components in addition to less power consumption, it is cheaper and cost effective.

\section{FUTURE APPLICATIONS OF LI-FI}

Li-Fi can be beneficial in many fields because of its various advantages and benefits as explained above. There are many applications that can use the Li-Fi technology in future. Some of them are listed below:

- Education System: In today's education system internet plays a major role but the basic problem using the available internet service is its speed, by using LiFi technology this problem can be solved to some extent as it provides high speed internet.

- Health Sector: With the advancement of technology, the medical sector is also using different methods to provide better medical facilities to the patients. Li-Fi can enhance and modernize this sector by providing internet facility in operation theaters, controlling medical equipments, organizing surgeries with robots and other automated events. The currently used networks like, Wi-Fi and others were not able to be used for services such as monitoring of apparatus in hospitals due to radiation concerns. The use of $\mathrm{Wi}-\mathrm{Fi}$ in hospitals is not allowed as it blocks the signals for monitoring equipments which may result in improper working of medical apparatus. So, Li-Fi solves all these problems and makes medical sector more technology friendly.

- Mobile Connectivity: Due to high speed connectivity and security provided by $\mathrm{Li}-\mathrm{Fi}$, it will become easy for any device such as mobile, laptop, tablets, etc. to connect to each other and communicate.

- Traffic Management: Li-Fi can also help in managing traffic resulting in its smooth flow and reduction in number of accidents. LED car lights can also alert drivers when other vehicles are too close.

- Disaster management: In case of natural calamities or natural disasters if people get to know about the protocols during that time, it will be more beneficial. In conditions like earthquakes and hurricane, Li-Fi can be 
used for emergency communication in subway stations, tunnels and common dead zones.

- Cheaper Internet in Aircrafts: Li-Fi by using LED reading bulb present inside airplanes provides a better, faster and cheaper means of communication to passengers travelling in an aircraft. As Wi-Fi may meddle with the navigational systems of the pilots.

- Other technology replacement: There are places where radio waves cannot be used. In such situations, the best alternative is Li-Fi because they don't use radio waves.

\section{LI-FI MARKET}

The demand for high speed and better connectivity of internet is increasing, so is the Li-Fi market especially in Asia Pacific. The Li-Fi market in U.S. in future is main market for $\mathrm{Li}-\mathrm{Fi}$ which is expected to introduce better aspects for $\mathrm{Li}-\mathrm{Fi}$ to grow at a higher rate. This demand for efficient and high speed wireless communication will result in the expansion of $\mathrm{Li}-\mathrm{Fi}$ market all over the world starting from the U.S. [7].

\section{FUTURE OF LI-FI}

As LED bulbs and lights are easily available source and are free to use, so in future there is a great scope for the evolution of Li-Fi technology on a larger level. As per this technology any and all LED bulbs can be used to transmit wireless data with better security, also it will lead to a cleaner and greener environment. This concept is spreading widely and is influencing many people as it is free and no license is required.

In near future, $\mathrm{Li}-\mathrm{Fi}$ can be one of the best and efficient alternative ways for high rate wireless data transmission. It can be an alternative to radio. So at the places where radio waves cannot be used, the use Li-Fi is the best such as virtual reality [6].

Recently, the researchers from University of Oxford have been engaged to achieve a bi-directional speed of 224 Gbps, which will allow downloading approximately 18 movies in a second. These speeds are much more than that offered by modern Wi-Fi [4].

\section{ACKNOWLEDGEMENT}

The authors are grateful to the people who motivated us to write this paper. It was a great feeling to write on such an interesting and trending topic "Light Fidelity: A way of data transmission". We have tried our best to represent the information gathered on this topic in best possible and easy form. We hope we have conveyed all the relevant information in the most understandable form.

\section{CONCLUSION}

This paper focuses on the brief details of Li-Fi technology. We have explained the working of Li-Fi and how it can be implemented. The benefits of Li-Fi over Wi-Fi are discussed. The different applications that can use Li-Fi in future are listed with brief explanation of the usage. Along with this the current status of Li-Fi in the market has also been inscribed with its future aspects.

\section{REFERENCES}

[1] Rahul R Sharma et al,’Li-Fi technology”, Int.J.Computer Technology \& Applications, Vol 5 (1),150-154.

[2] https://en.wikipedia.org/wiki/LED_lamp\#cite_ref-Nakamura_80 .

[3] Ashish Parnami1, Prof. Sanjiv Kumar, "A Review Paper on Light-Fidelity (Li-Fi)”, IJIRST , National Conference on Innovations in Micro-electronics, Signal Processing and Communication Technologies (V-IMPACT-2016) February 2016, ISSN: 2349-6010.

[4] Anurag Sarkar, Prof. Shalabh Agarwal, Dr. Asoke Nath, Li-Fi Technology: Data Transmission through Visible Light, ijarcsms, Volume 3, Issue 6, June 2015, ISSN: 2321-7782 (Online).

[5] https://www.techopedia.com/7/31772/technology-trends/whatare-the-advantages-and-disadvantages-of-Li-Fi-technology

[6] http://www.tec.gov.in/pdf/Studypaper/lifi\%20study\%20paper\%2 0-\%20approved.pdf

[7] https://www.transparencymarketresearch.com/lifi-market.html 